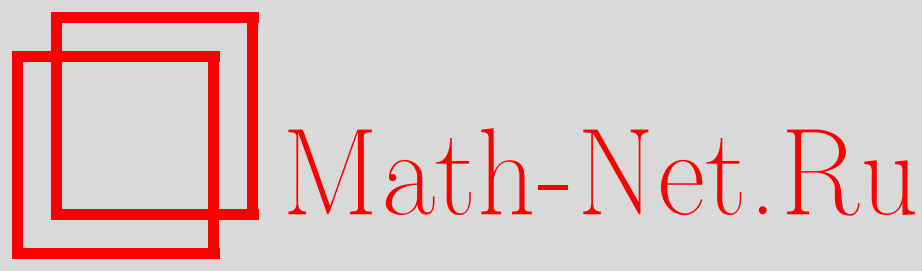

В. П. Маслов, Распределения Гиббса и Бозе-Эйнштейна для ансамбля самосопряженных операторов и в классической механике, ТМФ, 2008, том 155, номер 2, 312-316

DOI: https://doi.org/10.4213/tmf6213

Использование Общероссийского математического портала Math-Net.Ru подразумевает, что вы прочитали и согласны с пользовательским соглашением http://www . mathnet.ru/rus/agreement

Параметры загрузки:

IP : 52.23 .180 .231

26 апреля 2023 г., 15:44:48

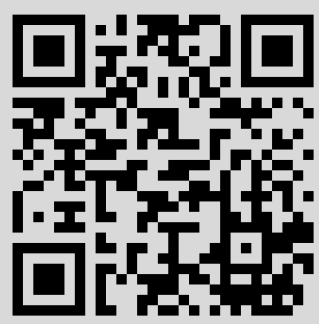




\title{
РАСПРЕДЕЛЕНИЯ ГИББСА И БОЗЕ-ЭЙНШТЕЙНА ДЛЯ АНСАМБЛЯ САМОСОПРЯЖЕННЫХ ОПЕРАТОРОВ И В КЛАССИЧЕСКОЙ МЕХАНИКЕ
}

\begin{abstract}
Вводится понятие ансамбля самосопряженных операторов и формулируются теоремы о связи чисел заполнения с числом собственных значений ансамбля. Формулируется теорема для гиббсовского распределения в классической механике.
\end{abstract}

Ключевые слова: распределение Гиббса, распределение Бозе-Эйнштейна, бозе-конденсат, упорядоченная и неупорядоченная выборка с возвращением, гиббсовский ансамбль.

В работе [1] о сверхтекучести классической жидкости в нанотрубках замечено, что "тяжелая" молекула (т.е. молекула, в которой числа протонов $k_{1}$ и нейтронов $k_{2}$ велики), содержащая четное число нейтронов, является классической, т.е. соотношение ее параметров таково, что можно полагать, что по сравнению с классическим действием константа Планка бесконечно мала в смысле нестандартного анализа. Кроме того, $3\left(2 k_{1}+k_{2}\right) N$-мерное уравнение Шредингера для $N$ молекул симметрично относительно перестановки молекул, а поскольку молекула состоит из четного числа фермионов, она является "тяжелой", т.е. классическим бозоном. В силу того, что молекула является классической, плотность распределения для системы $N$ невзаимодействующих частиц не удовлетворяет постулату (гипотезе) Гиббса о термодинамическом равновесии (см. [2], глава IV, формула (2.7)). А именно эта гипотеза лежит в основе предложенного Козловым доказательства распределения Гиббса. С другой стороны, эргодическая гипотеза, как показано Козловым [2], также не выдерживает критики (в частности, опровергается теорией KAM). Мы опишем здесь другой подход к распределению Гиббса, не основанный на гипотезах. Мы применим этот подход к распределению классических бозонов.

В работе [3] была рассмотрена система уравнений квантового осциллятора. Однако полученные оценки без труда переносятся на достаточно общие операторы Шредингера. Это позволяет доказать приведенные ниже теоремы.

Рассмотрим самосопряженный оператор $\widehat{H}$ с дискретным положительным спектром, $\lambda_{1} \leqslant \lambda_{2} \leqslant \cdots \leqslant \lambda_{n} \cdots, \lambda_{n} \rightarrow \infty$ при $n \rightarrow \infty$, и соответствующие собственные

* Московский государственный университет им. М. В. Ломоносова, Москва, Россия. E-mail: v.p.maslov@mail.ru 
подпространства $P_{1}, P_{2}, \ldots, P_{n}, \ldots$ в гильбертовом пространстве $L_{2}=L_{2}\left(\mathbb{R}^{k}\right)$, где $k$ - любое конечное целое число. Будем предполагать, что для числа собственных значений, меньших заданного $E$ при $E \rightarrow \infty$, выполняется правило Вейля (физики говорят, что число собственных значений пропорционально фазовому объему). Иногда эту формулу называют формулой Куранта.

Рассмотрим тензорное произведение $N$ гильбертовых пространств

$$
\mathcal{L}_{2}=\underbrace{L_{2}\left(\mathbb{R}^{k}\right) \otimes L_{2}\left(\mathbb{R}^{k}\right) \otimes L_{2}\left(\mathbb{R}^{k}\right) \cdots \otimes L_{2}\left(\mathbb{R}^{k}\right)}_{N \text { сомножителей }},
$$

где $N \rightarrow \infty$, и определим на нем оператор

$$
\widehat{H}_{N}=\underbrace{[\widehat{H} \otimes 1 \otimes 1 \cdots \otimes 1]+[1 \otimes \widehat{H} \otimes 1 \cdots \otimes 1]+\cdots+[1 \otimes 1 \cdots 1 \otimes \widehat{H}]}_{N \text { сомножителей }} .
$$

Запишем уравнение

$$
i \frac{\partial \Psi}{\partial t}=\widehat{H} \Psi
$$

с начальными условиями вида $\left.\Psi\right|_{t=0}=\varphi_{s}^{(i)}(x), x \in \mathbb{R}^{k}, \varphi_{s}^{(i)}(x) \in L_{2}\left(\mathbb{R}^{k}\right), i=$ $1,2, \ldots, N$, такими, что последовательность $\varphi_{s}^{(i)}(x)$ при $s \rightarrow \infty$ слабо сходится к нулю, $\left\|\varphi_{s}^{(i)}(x)\right\|_{L_{2}\left(\mathbb{R}^{k}\right)}=1$, а $E_{s}^{(i)}=\left(\varphi_{s}^{(i)}(x), \widehat{H} \varphi_{s}^{(i)}(x)\right) \rightarrow \infty$ при $n \rightarrow \infty, E_{s}^{(i)} \leqslant E$, $P_{n} \varphi_{s}^{(i)}=0$, если $\lambda_{n}>E$. Соответственно, решения уравнения

$$
i \frac{\partial \Psi_{N}}{\partial t}=\widehat{H}_{N} \Psi_{N}, \quad \Psi_{N} \in \mathcal{L}_{2}
$$

с начальным условием вида

$$
\left.\Psi_{N}\right|_{t=0}=\varphi_{s}^{(1)}\left(x_{1}\right) \varphi_{s}^{(2)}\left(x_{2}\right) \ldots \varphi_{s}^{(N)}\left(x_{N}\right), \quad x_{i} \in \mathbb{R}^{n}
$$

при $N \rightarrow \infty, s \rightarrow \infty$ будем называть гиббсовским ансамблем.

Если $\varphi_{s}^{(k)}\left(x_{k}\right)$ не равны друг другу при $k=1,2, \ldots, N$, то мы определим параметр $b_{E}$ из условия

$$
\frac{\operatorname{Sp}\left(\widehat{H} e^{-b_{E} \widehat{H}}\right)}{\operatorname{Sp}\left(e^{-b_{E} \widehat{H}}\right)}=E .
$$

Обозначим $N_{0}=\mathrm{Sp} e^{-b_{E} \widehat{H}}$. Числа заполнения оператора $\widehat{H}_{N}$, отвечающие собственной функции $\Psi_{i}$ оператора $\widehat{H}$, меньшие или равные $E N$, будем обозначать $N_{i}$. Сумму $N_{i}+N_{i+1}+\cdots+N_{i+k}$ обозначим через $N_{i}^{(k)}$ - числа заполнения ${ }^{1)}$, отвечающие набору функций $\Psi_{i}, \Psi_{i+1}, \ldots, \Psi_{i+n}$, а проектор на подпространство, натянутое на собственные подпространства, отвечающие им, - через $P_{i}^{(k)}$.

Для несимметричного гиббсовского ансамбля имеет место следующая теорема.

Теорема 1. Существуют такие постоянные $C_{l}, l=1,2, \ldots$, что для любых $i>1$ и $n>0$ числа заполнений $N_{i}^{(n)}$ удовлетворяют неравенству

$$
\mathrm{P}\left(\left|N_{i}^{(n)}-B \operatorname{Sp}\left(P_{i}^{(n)} e^{-b_{E} \widehat{H}}\right)\right|>B \sqrt{N_{0} \ln N_{0}} \psi\left(N_{0}\right)\right) \leqslant C_{l} N_{0}^{-l}, \quad l=1,2, \ldots,
$$

где $B=N / N_{0}, \psi(x)$ - положительная функиия, произвольно медленно стремящаяся $\kappa+\infty$ при $x \rightarrow+\infty$, a $\mathrm{P}(\cdot)$ - отношение числа собственных значений гамильтониана $\widehat{H}_{N}$, не превосходящих $N E$ и удовлетворяющих стоящему в скобках

1) Для определения числа $N_{i}^{(k)}$, физический смысл которого есть число частиц, можно считать частицы неразличимыми. 
неравенству, к общему числу собственных значений гамильтониана $\widehat{H}_{N}$, не превосходящих NE (плотность спектра) $)^{2)}$.

Если начальное условие (3) симметрично (т.е. $\varphi_{s}^{(k)}(x)=\varphi_{s}^{(m)}(x)$ при всех $k$ и $m$ ), то оно разлагается только по симметрическим собственным функциям оператора $\widehat{H}_{N}$. Выражение вида $\sum_{i=1}^{n} \lambda_{i} N_{i}$ для любых $N_{i}$, удовлетворяющих условию $\sum_{i=1}^{n} N_{i} \leqslant N$, есть собственное значение $\widehat{H}_{N}$, отвечающее симметричным по $\left(x_{i}, x_{j}\right)$ собственным функциям, а значит, их число равно $N_{i}^{(n)}$. Будем называть решения этой задачи симметричным гиббсовским ансамблем. Поскольку $\sum \lambda_{i} N_{i}$ является собственным значением оператора ${ }^{3)} \widehat{H}_{N}$, отвечающим симметрическим собственным функциям, то $\sum N_{i}$ по всем $N_{i}$ является числом всех собственных значений оператора $\widehat{H}_{N}$, меньших, чем $E N$.

Пусть $b_{E, N}$ - решение уравнения

$$
\operatorname{Sp}\left(\frac{\widehat{H}}{e^{b_{E, N} \widehat{H}}-1}\right)=E \operatorname{Sp}\left(\frac{1}{e^{b_{E, N} \widehat{H}}-1}\right)+\lambda_{1}\left(N-\operatorname{Sp}\left(\frac{1}{e^{b_{E, N} \widehat{H}}-1}\right)\right) .
$$

Тогда для "почти всех" чисел заполнения $N_{i}^{(n)}$ при $n \rightarrow \infty$ имеет место равенство

$$
N_{i}^{(n)} \cong \operatorname{Sp}\left(P_{i}^{(n)} \frac{1}{e^{b_{E, N} \widehat{H}}-1}\right),
$$

где $P_{i}^{(n)}=P_{i}+P_{i+1}+\cdots+P_{i+n}-$ проектор на подпространство, порожденное $P_{i} L_{2}, P_{i+1} L_{2}, \ldots, P_{i+n} L_{2}$.

Более точно утверждение формулируется следующим образом.

Теорема 2. Существуют такие постоянные $C_{l}, l=1,2, \ldots$, что для любых $i>1$ и $n>0$ числа заполнения $N_{i}^{(n)}$ удовлетворяют неравенству

$$
\mathrm{P}\left(\left|N_{i}^{(n)}-\operatorname{Sp}\left(P_{i}^{(n)} \frac{1}{e^{b_{E, N} \hat{H}}-1}\right)\right|>\sqrt{\widetilde{N}_{0} \ln \widetilde{N}_{0}} \psi\left(N_{0}\right)\right) \leqslant C_{l} \widetilde{N}_{0}^{-l}, \quad l=1,2, \ldots,
$$

где

$$
\widetilde{N}_{0}=\operatorname{Sp}\left(\frac{1}{e^{b_{E, N} \widehat{H}}-1}\right)
$$

$\psi(x)$ - положительная функиия, произвольно медленно стремящаяся $\kappa+\infty$ при $x \rightarrow+\infty$, a $\mathrm{P}(\cdot)$ - отношение числа собственных значений гамильтониана $\widehat{H}_{N}$, отвечающих симметричным по $\left(x_{i}, x_{j}\right)$ собственным функииям, не превосходящих NE и удовлетворяющих стоящему в скобках неравенству, к общему числу собственных значений гамильтониана $\widehat{H}_{N}$, не превосходящих $N E$ и отвечающих симметрическим собственным функииям.

Таким образом, в симметрическом случае $\widetilde{N}_{0}$ зависит от $N$, и если $\widetilde{N}_{0}(N)<N$, то возникает "диффузия", при которой увеличивается кратность $\lambda_{1}$ на величину $N-$ $\widetilde{N}_{0}(N)$, т.е. при $N \gg \widetilde{N}_{0}(N)$ возникает скопление собственных значений оператора $\widehat{H}_{N}$ на нижнем уровне $\lambda_{1}$ (конденсат).

\footnotetext{
${ }^{2)} \mathrm{B}$ книге [4] (с. 44) для микроканонического распределения употребляется термин "густота спектра ".

3) В работе [3] использовался термин “число вариантов", который определялся как число собственных значений оператора $\widehat{H}_{N}$.
} 
Теоремы 1 и 2 означают, что если мы задаем $N_{i}^{(n)}$ на интервале $\lambda_{i}, \ldots, \lambda_{i+n}$ и считаем отношение числа собственных значений с заданными $N_{i}, N_{i+1}, \ldots, N_{i+n}$ и остальными произвольными значениями $N_{j}$ при $j<i, j>i+n$ к общему числу собственных значений оператора $\widehat{H}_{N}$, не превосходящих $E N$, то это отношение стремится к нулю как $N_{0}^{-s}$ для любого $s$ вне интервала

$$
N_{i}^{(n)} \sim \operatorname{Sp}\left(P_{i}^{(n)} e^{-b_{E} \widehat{H}}\right) \pm B \sqrt{N_{0} \ln N_{0}} \psi\left(N_{0}\right)
$$

для теоремы 1 или соответствующего интервала для теоремы 2. Можно сказать, что речь идет о плотности спектра гиббсовского ансамбля и о том, что, в отличие от точки зрения физиков относительно того, что формула (4) дает среднее значение энергии, она определяет значение $E N$, ограничивающее плотность спектра оператора $\widehat{H}_{N}$. Можно также сказать, что это общее число собственных значений оператора $\widehat{H}_{N} P_{E N}$, где $P_{E N}$ - проектор на все собственные подпространства, отвечающие всем $\mu_{n}^{(N)} \leqslant E N$, где $\mu_{n}^{(N)}$ - все собственные значения оператора $\widehat{H}_{N}$, не большие, чем $N E$.

Пусть $\mathcal{P}_{i}^{n}-$ проектор на все собственные подпространства оператора $P_{E N} \widehat{H}_{N}$, лежащие вне интервала, окружающего собственные значения оператора $P_{i}^{n} \widehat{H} e^{-b_{E} \widehat{H}} \pm$ $B \sqrt{N_{0} \ln N_{0}} \psi\left(N_{0}\right)$. Тогда отношение числа собственных значений оператора $\mathcal{P}_{i}^{n} \widehat{H}_{N}$ к числу собственных значений оператора $P_{E N} \widehat{H}_{N}$ стремится к нулю быстрее любой степени $N_{0}^{-1}$.

Перейдем теперь к классическому распределению Бозе-Эйнштейна. Пусть гамильтониан $H(p, q)$ есть выпуклая функция, растущая не медленнее, чем $|p|+|q|$, и не быстрее, чем $|p|^{s}+|q|^{s}$, где $s$ - любое целое число, а при $p \rightarrow 0, q \rightarrow 0$ стремящаяся к нулю не медленнее, чем $\bar{p}^{2}$. Пусть $N$ - число частиц. Найдем $b$ из соотношения

$$
\int\left(e^{b H(p, q)}-1\right)^{-1} d p d q=N
$$

Пусть $\rho_{E_{l}}^{\Delta}-$ плотность числа частиц в интервале $E_{l}$ и $E_{l+1}$, где $\Delta=E_{l+1}-E_{l}$. Дадим более точное определение.

Разобьем фазовое пространство $p, q$ на конечное число областей $E_{l} \leqslant H(p, q) \leqslant$ $E_{l+1}, l=0, \ldots, s-1, E_{0}=0, E_{s}=E, p \in \mathbb{R}^{n}, q \in \mathbb{R}^{n}$, и аналогичным образом фазовое пространство $\mathbb{R}^{2 n N}$ разобьем на соответствующие области. Производя неупорядоченную выборку с возвращением $N_{l}$ из разбиения областей пространства $\mathbb{R}^{2 n N}$ в "ящик" $E_{l} \leqslant H(p, q) \leqslant E_{l+1}$ при условии

$$
\sum_{i=1}^{N} H\left(p_{i}, q_{i}\right) \leqslant N E
$$

где $E=N^{-1} \int H(p, q)\left(e^{b H(p, q)}-1\right)^{-1} d p d q$, мы получим $\rho_{E_{l}}^{\Delta}=N_{l} / N[5]$. В приведенной ниже теореме $\mathrm{P}$ есть лебегова мера фазового объема, определенного в скобках в (10), относительно всего объема (9).

Теорема 3. Справедливо соотношение

$$
\mathrm{P}\left(\left|N \rho_{E_{l}}^{\Delta}-\int_{E_{l} \leqslant H(p, q) \leqslant E_{l+1}}\left(e^{b H(p, q)}-1\right)^{-1} d p d q\right| \geqslant \sqrt{N \ln N} \psi(N)\right)=O\left(N^{-m}\right),
$$

где $m$ - любое иелое число. 
Отметим, что уравнения для “одетого" потенциала в случае распределения БозеЭйнштейна в классической механике принимают вид

$$
\bar{u}(q)=u(q)+\int \frac{V\left(q-q^{\prime}\right) d p^{\prime} d q^{\prime}}{\exp \left\{b^{-1}\left(\left|p^{\prime}\right|^{2} /(2 m)+\bar{u}\left(q^{\prime}\right)\right)\right\}-1},
$$

где $u(q)$ - внешнее поле, а $V(x-y)$ - потенциал парного взаимодействия.

Последнее уравнение не было известно в физике. Его впервые получил автор для общего случая в серии статей [6]. Для ферми-системы известно температурное уравнение Томаса-Ферми [7].

Рассмотрим при тех же условиях, что и выше, упорядоченную выборку. С физической точки зрения это означает, что рассматривается $N$ различимых частиц, а $\rho_{E_{l}}^{\Delta}$ снова обозначает "плотность" частиц в интервале энергии $E_{l} \leqslant H(p, q) \leqslant E_{l+\Delta}$. В этом случае при $n=3$ имеет место распределение Гиббса.

Пусть выполнены вышеприведенные условия на функцию $H(p, q)$. Пусть задано $N$. Определим $b$ из условия

$$
\int_{0}^{\infty} e^{-b H(p, q)} d p d q=N
$$

Определим $E$ в $(9)$ как $N^{-1} \int_{0}^{\infty} H(p, q) e^{-b H(p, q)} d p d q$. Тогда будет иметь место следующая теорема.

Теорема 4. Имеет место соотношение

$$
\mathrm{P}\left(\left|N \rho_{E_{l}}^{\Delta}-\int_{E_{l} \leqslant H(p, q) \leqslant E_{l+1}} e^{-b H(p, q)} d p d q\right| \geqslant \sqrt{N \ln N} \psi(N)\right)=O\left(N^{-m}\right),
$$

где $m$ - любое целое число.

Вероятность Р здесь также есть лебегова мера фазового объема, определенного в скобках (13), относительно всего фазового объема (9).

Благодарности. Автор приносит глубокую благодарность В. Е. Назайкинскому за плодотворные обсуждения и В. Е. Подольскому за полезные консультации.

\section{Список литературы}

[1] В. П. Маслов, ТМФ, 153:3 (2007), 388-408.

[2] В.В. Козлов, Тепловое равновесие по Гиббсу и Пуанкаре, Ин-т компьютерных исследований, М.-Ижевск, 2002.

[3] В. П. Маслов, ТМФ, 154:1 (2008), 207-208.

[4] Л. Д. Ландау, Е. М. Лифшиц, Теоретическая физика Т. V. Статистическая физика, Наука, М., 1964.

[5] А.Н. Ширяев, Вероятностъ, т. 1, МЦНМО, М., 2004.

[6] V.P. Maslov, Russ. J. Math. Phys., 2:4 (1994), 527-534; 3:1 (1995), 123-132; 271-276; 401-406; 529-534; 4:1 (1996), 117-122; № 2, 265-270; № 4, 539-546; 5:4 (1997), 473-488; № 1, 123-130; № 2, 273-278; № 3, 405-412; № 4, 529-534.

[7] Н. Ашкрофт, Н. Мермин, Физика твердого тела, т. 1, Мир, М., 1979. 